\begin{tabular}{|c|l|}
\hline Title & $\begin{array}{l}\text { Samia cynthiaversus Bombyx mori : Comparative gene mapping between a species with a low-number karyotype and } \\
\text { the model species of Lepidoptera }\end{array}$ \\
\hline Author(s) & Yoshido, A tsuo; Y asukochi, Yuji; Sahara, Ken \\
\hline Citation & $\begin{array}{l}\text { Insect Biochemistry and Molecular Biology, 41(6), 370-377 } \\
\text { https://doi.org/A0.1016/.ibmb.2011.02.005 }\end{array}$ \\
\hline Issue Date & 2011-06 \\
\hline Doc URL & http://hdl.handle.net/2115/45607 \\
\hline Type & article (author version) \\
\hline Additional Information & There are other files related to this item in HUSCAP. Check the above URL. \\
\hline File Information & IBMB41-6_370-377.pdf \\
\hline
\end{tabular}

Instructions for use 


\section{Samia cynthia versus Bombyx mori: Comparative gene mapping between a species with a low-number karyotype and the model species}

\section{of Lepidoptera.}

Atsuo Yoshido ${ }^{\mathrm{a}^{*}}$, Yuji Yasukochi ${ }^{\mathrm{b}}$, Ken Sahara $^{\mathrm{a}}$

${ }^{a}$ Laboratory of Applied Molecular Entomology, Graduate School of Agriculture, Hokkaido University, N9, W9, Kita-ku, Sapporo 060-8589, Japan

${ }^{\mathrm{b}}$ Insect Genome Research Unit, National Institute of Agrobiological Sciences, Tsukuba, Ibaraki 305-8634, Japan

Running title: Gene-based mapping in Samia cynthia

Key words: comparative gene mapping; fosmid-FISH; Lepidoptera; sex chromosome;

conserved synteny.

"corresponding author: Atsuo Yoshido

Laboratory of Applied Molecular Entomology, Graduate School of Agriculture,

Hokkaido University, N9, W9, Kita-ku, Sapporo 060-8589, Japan

E-mail: yoshido@abs.agr.hokudai.ac.jp ～Phone \& Fax: +81-11-706-2402 


\section{Abstract}

We performed gene-based comparative FISH mapping between a wild silkmoth, Samia cynthia ssp. with a low number of chromosomes $(2 n=25-28)$ and the model species, Bombyx mori $(2 \mathrm{n}=56)$, in order to identify the genomic components that make up the chromosomes in a low-number karyotype. Mapping of 64 fosmid probes containing orthologs of B. mori genes revealed that the homologues of either two or four B. mori chromosomes constitute the S. c. ricini (Vietnam population, $2 \mathrm{n}=27 \bigcirc / 28 \AA, \mathrm{Z} 0 / \mathrm{ZZ}$ ) autosomes. Where tested, even the gene order was conserved between $S$. c. ricini and $B$. mori. This was also true for the originally autosomal parts of the neo-sex chromosomes in S. c. walkeri (Sapporo population, $2 \mathrm{n}=26 \propto+26 \hat{\jmath}$, neo-Wneo-Z/neo-Zneo-Z) and $S$. cynthia subsp. indet. (Nagano population, $2 \mathrm{n}=25 \mathrm{O} / 26{ }^{\lambda}$, neo- $-\mathrm{WZ}_{1} \mathrm{Z}_{2} / \mathrm{Z}_{1} \mathrm{Z}_{1} \mathrm{Z}_{2} \mathrm{Z}_{2}$ ). The results are evidence for an internal stability of lepidopteran chromosomes even when all autosomes had undergone fusion processes to form a low-number karyotype. 


\section{Introduction}

Genome rearrangements among species have been recently revealed by comparative genomic analyses. These showed that mammalian genomes have changed by reshuffling chromosomal segments from the common ancestral karyotype (Ferguson-Smith and Trifonov, 2007), while birds show a high degree of collinearity among species (Ellegren, 2010). In insects, comparative genome analysis among genome-sequenced 12 Drosophila species revealed that many orthologous genes mapped to the corresponding chromosomal arms but gene orders were scrambled between species (Ranz et al., 2001; Bhutkar et al., 2008). The availability of fully sequenced genomes provides an opportunity to study chromosomal rearrangements and evolutionary relationship among related species in detail.

Lepidoptera, moths and butterflies, consist of more than 150,000 species. They are the second largest order of animals (Kristensen and Skalski, 1999; Grimaldi and Engel, 2005). Lepidoptera have holokinetic chromosomes like aphids and bugs, and share the sex chromosome system of female heterogamety with caddis flies (Trichoptera) (Traut et al., 2007). The most common chromosome numbers of Lepidoptera range from $\mathrm{n}=28$ to $\mathrm{n}=32$ among the more than 1,000 species investigated 
(Robinson, 1971). There are, however, also species with lower or higher number karyotypes in Lepidoptera. Species with low or high-number karyotypes are thought to have evolved by chromosomal fusion and fission from the putative ancestor with $n=31$ chromosome number (Lukhtanov, 2000; Marec et al., 2010).

Since the first lepidopteran genome, that of Bombyx mori, has been accessible in public databases, comparative mapping of genes was carried out against B. mori $(n=28)$ by either linkage analysis (Beldade et al., 2009) or BAC-FISH (Yasukochi et al., 2009). These authors detected a high degree of conserved synteny and gene order between $B$. mori and two other species with the same chromosome number, Bicyclus anynana and Manduca sexta. Pringle et al. (2007) detected conserved gene order in Heliconius melpomene and suggested simple fusion events to account for the reduced chromosome number $(\mathrm{n}=21)$ of this species. These studies suggested an internal stability of lepidopteran chromosomes. DNA sequencing of each 15 selected BACs from Helicoverpa armigera and Spodoptera frugiperda also revealed a high degree of conserved synteny with only a few rearrangements between $B$. mori and the two noctuid moths, both of them having the supposed ancestral chromosome number $(n=31)$ of Lepidoptera (d'Alençon et al., 2010).

Samia cynthia, a wild silkmoth, belongs to the family Saturniidae which is 
closest to the family Bombycidae including B. mori. S. cynthia has a reduced chromosome number, ranging from $2 \mathrm{n}=25$ to $2 \mathrm{n}=28$ and is about half that of Bombyx mori. The variation is due to the variable sex chromosome constitution among geographic subspecies (Yoshido et al., 2005b). Previous study proposed repeated autosome-sex chromosome fusions resulted in the variable sex chromosome constitution as found in S. cynthia subspecies (Yoshido et al., 2010). The sex chromosome constitution in S. c. walkeri (the ailanthus silkworm, Sapporo population) females is designated as neo- $\mathrm{W}$ and neo- $\mathrm{Z}$ chromosomes, which originated by fusion of the ancestral $\mathrm{W}$ and $\mathrm{Z}$ with an autosome pair $\left(\mathrm{A}_{1}\right)$. Then sex chromosome constitution (designated as neo- $\mathrm{WZ}_{1} \mathrm{Z}_{2}$ ) in $S$. cynthia subsp. indet. (the shinju silkworm, Nagano population) females has been formed by next evolutionary step, in which neo-W chromosome fused with an autosome $\left(\mathrm{A}_{2}\right)$ and, consequently, its unfused homologue became a $\mathrm{Z}_{2}$ chromosome. In S. c. ricini (the Eri silkworm, Vietnam population), no such fusion of sex chromosomes with autosomes occurred and, the sex chromosome constitution in females is $\mathrm{Z} 0$, which arose from ancestral WZ by a loss of the W chromosome. Hence, the chromosome number is $2 \mathrm{n}=27 / 28$ in $S$. c. ricini with a $\mathrm{Z0} / \mathrm{ZZ}$, $2 n=26 / 26$ in S. c. walkeri with neo-Wneo-Z/neo-Zneo-Z, and $2 n=25 / 26$ in S. cynthia subsp. indet. with neo- $\mathrm{WZ}_{1} \mathrm{Z}_{2} / \mathrm{Z}_{1} \mathrm{Z}_{1} \mathrm{Z}_{2} \mathrm{Z}_{2}$ sex chromosomes in female/males. 
We show here by comparative gene mapping between $S$. cynthia subspecies and B. mori the internal stability of lepidopteran chromosomes even when low-chromosome-number karyotypes evolve by chromosome fusion. We constructed a fosmid-library of S. cynthia and carried out gene-based comparative FISH mapping between $B$. mori and the three S. cynthia subspecies. Sixty-four fosmid probes which contain orthologs of B. mori genes, cytogenetically identified all chromosomes of the $S$. c. ricini. Furthermore, fosmid-FISH mapping identified the gene order of the neo-sex chromosomes in S. c. walkeri and S. cynthia subsp. indet.

\section{Materials and Methods}

\subsection{Insects}

Samia cynthia was originally collected at three different locations: S. c. walkeri in Sapporo, Japan, S. cynthia subsp. indet. in Nagano, Japan, and S. c. ricini in Vietnam

(for details, see Yoshido et al., 2005b). For rearing, we released the hatched larvae on an Ailanthus altissima tree in a field of the Field Science Center for Northern Biosphere, Hokkaido University (Sapporo, Japan). 


\subsection{Construction of a Samia cynthia fosmid library}

A fosmid library was constructed from female pupae of S. c. walkeri (Sapporo population). High molecular weight DNA was physically sheared and fractionated by pulsed-filed gel electrophoresis. Blunt-ended DNA fragments collected from the 33 to $48 \mathrm{~kb}$ fraction were cloned into pCC1FOS vector. The ligates were packaged into Escherichia coli (EPI300) by in vitro packaging kit of MaxPlax Lambda packaging Extract (EPICENTER, Madison, WI, USA). A total of 35,712 single colonies were picked up and stocked into 93 of 384-well microplates.

\subsection{Fosmid screening for FISH mapping}

Sequence information of S. cynthia orthologs of B. mori genes was acquired mainly from the S. c. ricini EST database (http://silkbase.ab.a.u-tokyo.ac.jp/cgi-bin/index.cgi) (Arunkumar et al., 2008) and NCBI Genbank. Because the orthologs of BR-C, laccase2, PKGIb and egg specific protein have not yet been recorded in any public database, we amplified segments of them by DOP-PCR. Degenerated oligonucleotide primers (Table S1) were designed from regions conserved between B. mori and either Drosophila melanogaster and/or other lepidopteran species. The amplified fragments from S. $c$. 
walkeri genomic DNA were cloned into pGEM T-easy vector (Promega KK, Tokyo, Japan) and sequenced with an ABI PRISM3100 Genetic Analyzer (Applied Biosystems, Tokyo, Japan) according to the protocol recommended by the manufacturer. Tblastn searches by KaikoBlast (http://sgp.dna.affrc.go.jp/KAIKObase/) showed $81.25 \%$, $73.43 \%, 98.48 \%$ and $58.38 \%$ identities for the orthologs of BR-C, laccase2, $P K G-I b$ and egg specific protein respectively.

We then designed sequence-tagged site (STS) primers (Table S1) in S. c. walkeri in order to isolate clones carrying the respective orthologs from the fosmid library. For the isolation we followed the 3-step method described by Yasukochi (2002). Briefly, the first PCR screening was done against DNA pools from 93 plates, a mixture of 384 fosmids from each plate. The second step was applied only to positive plates with the same size of PCR products as from genomic DNA. We performed PCRs against fosmid-DNA pools from 24 columns and 16 rows to determine the coordinates of the positive clone. In the last step, we individually repeated the PCR to confirm the positive selection. We also confirmed the positive selection by sequencing the PCR products. 


\subsection{Chromosome preparations}

Spread chromosome preparations from females and males were obtained according to methods described in Sahara et al. (1999) and Yoshido et al. (2005b). Briefly, gonads of the last instar larvae were dissected in a saline solution and fixed for $10-15 \mathrm{~min}$ in Carnoy's fixative (ethanol, chloroform, acetic acid, 6:3:1). Testes but not ovaries were swollen for $10-15 \mathrm{~min}$ in a hypotonic solution $(75 \mathrm{mM} \mathrm{KCl})$ before fixation. Cells were dissociated in $60 \%$ acetic acid and spread on a heating plate at $50^{\circ} \mathrm{C}$. Then preparations were passed through a graded ethanol series $(70 \%, 80 \%$ and $98 \%)$ and stored at $-30^{\circ} \mathrm{C}$ until further use.

\subsection{Fosmid-FISH mapping}

Fosmid-FISH was carried out according to the BAC-FISH method described in Yoshido et al. (2005a) with slight modifications. Fosmid-DNA was extracted with a Plasmid Midi kit (Qiagen GmbH, Hilden, Germany). DNA labeling was done by nick translation using Nick Translation Mix (Roche Diagnostics GmbH, Mannheim, Germany) with Green-dUTP, Orange-dUTP, and Red-dUTP (Abbott Molecular Inc., Des Plaines, IL, USA), and Cy5-dUTP (GE Healthcare UK, Buckinghamshire, UK). We used the reprobing protocol for Lepidoptera (Shibata et al., 2009) for karyotyping and, if more 
than four fosmid probes were mapped to a single chromosome. The S. cynthia ortholog of RpL4 was recovered by PCR with primers devised from the EST database (SilkBase) because we could not isolate a suitable fosmid clone in our library. Similarly, $18 \mathrm{~S}$ rDNA was recovered by PCR. To prepare FISH probes, both were labeled with Orange-dUTP by PCR according to the method described in Yoshido et al. (2010).

Denaturation of preparations was carried out at $72^{\circ} \mathrm{C}$ for $3.5 \mathrm{~min}$ in $70 \%$ formamide, $2 \times \mathrm{SSC}$, after the slides were passed through an ethanol series and air-dried. For one preparation, we used a probe cocktail with $0.1-0.4 \mu \mathrm{g}$ each labeled DNA (see

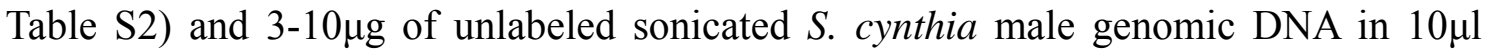
hybridization solution ( $50 \%$ formamide, $10 \%$ dextran sulfate, $2 \times \mathrm{SSC}$ ). Hybridization in a moist chamber at $37^{\circ} \mathrm{C}$ for 3 days was followed by washing at $62^{\circ} \mathrm{C}$ in $0.1 \times \mathrm{SSC}, 1 \%$ Triton X-100. The slides were counterstained and mounted in $30 \mu 1$ VECTORSHIELD with DAPI (Vector Laboratories, Inc., Burlingame, USA). Counterstained chromosome and hybridization signals were captured with a DFC350FX B\&W CCD camera (Leica Microsystems Inc., Tokyo, Japan) described in Sahara et al. (2007). The signal and chromosome images were processed by Adobe Photoshop according to Yoshido et al. (2005a). 


\section{Results}

\subsection{Isolation of $S$. cynthia fosmid clones containing orthologs of $B$. mori genes}

We isolated a total of 71 fosmid clones by PCR-based screening using 66 STS primer sets (Table S1). The fosmid clones carrying the orthologs of B. mori genes were selected from each of the 27 B. mori autosomes and the $\mathrm{Z}$ chromosome (Table 1).

\subsection{Identification of individual chromosomes in S. cynthia ricini}

To identify individual bivalents in S. c. ricini, we carried out FISH mapping on pachytene nuclei using the S. c. walkeri fosmid probes. In total, 64 fosmid clones mapped to single locations of S. c. ricini chromosomes (Fig. 1, Table 1). Although the clones used were from S. c. walkeri, the hybridization signals were strong enough in the other S. cynthia subspecies. This enabled us to identify the 13 autosomal bivalents and the $\mathrm{Z}$ chromosome of the S. c. ricini karyotype (Fig. 1) and their correspondence to $B$. mori (Table 1). Three of the fosmid probes produced multiple signals (Table S2) and, hence were discarded from further experiments.

The $\mathrm{Z}$ chromosome of $S$. c. ricini was recognized only by probes which 
contained orthologs of B. mori Z chromosomal genes. Of the 13 S. c. ricini autosomes, ten corresponded to two B. mori different autosomes each (Fig. 1, Table 1). There were three exceptions. One was chromosome 1 of $S$. c. ricini which corresponded to four $B$. mori chromosomes, 2, 20, 26, and 27. The others were chromosomes 11 and 12. Chromosome 11 of S. c. ricini corresponded to B. mori chromosome 24 and a part of $B$. mori chromosome 11. S. c. ricini chromosome 12 corresponded to B. mori chromosome 21 and the other part of $B$. mori chromosome 11. In those cases where we used more than two probes (B. mori chromosomes 8,11,12, 15 and 19), even the gene order was conserved between the component parts of S. c. ricini chromosomes and the B. mori chromosomes. We confirmed that S. c. ricini chromosome 11 was the chromosome carrying nucleolar organizer region (NOR) by mapping the $18 \mathrm{~S}$ rDNA fragment to that chromosome (Fig. 1). Our previous data of B. mori BAC-FISH revealed that the B. mori NOR located somewhere between RpL4 and Topo II (Yoshido et al., 2010). In S. c. ricini, however, the signal of $18 \mathrm{~S}$ rDNA (white signal of Chr11 in Fig. 1) was detected in the region between the $R p L 4$ ortholog and the $P 450$ ortholog. The result reveals that a limited repositioning of the NOR has occurred in the B. mori or S. c. ricini phylogenetic lineages. 


\subsection{Fosmid-FISH karyotype of Samia cynthia}

For karyotyping, i. e. identifying all chromosomes of a set, we used a total of 22 fosmid probes (Fig. 2, Table S2). We identified 11 autosomal bivalents (Chromosome 2-10, 12 and 13) in a first round of FISH. They were distinguished by the color combination of the probes, or by the different signal position when they had the same color combination (chromosome 2 and 6, 3 and 5, 9 and 12). To recognize the remaining chromosomes 1, 11 and $\mathrm{Z}$, we reprobed the slides in a second round of FISH. In addition, we used two probes for chromosomes 2 and 3 to confirm results of the first round. In this manner, we identified all 13 autosomal bivalents and the $\mathrm{Z}$ univalent, and hence definitively karyotyped the S. c. ricini pachytene complement (Fig. 2).

\subsection{Sex chromosome variation in $S$. cynthia subspecies}

We also carried out fosmid-FISH mapping in the other two S. cynthia subspecies, S. c. walkeri (Sapporo population) and S. cynthia subsp. indet. (Nagano population). The homologue of S. c. ricini chromosome 13 forms the neo-sex chromosomes (the segment of autosomal origin) of S. c. walkeri and S. cynthia subsp. indet. (Fig. 3b, c). In $S$. cynthia subsp. indet., the homologue of S. c. ricini and S. c. walkeri chromosome 12 contribute an additional segment to the neo-W and the $Z_{2}$ chromosome (Fig. 3a, c). The 
results confirm the step-by-step evolution of sex chromosome recently proposed by Yoshido et al. (2010) in these S. cynthia subspecies. Our results also show that the gene order is strictly conserved between the respective segments of autosomal origin in neo-sex chromosomes from the Japanese subspecies of S. cynthia and the homologous autosomes of S. c. ricini and B. mori (compare Figs. 1 and 3).

\section{Discussion}

We show here that each chromosome of S. cynthia can be reliably identified by FISH, using fosmid probes. A selected set of 22 probes from a genomic fosmid library was sufficient to karyotype S. cynthia (Fig. 2). Fosmid clones have been used as reliable cytogenetical markers for FISH karyotyping in animals and plants (Zhang et al., 2008; Dalzell et al., 2009; Liu et al., 2010). However, this is the first application of fosmid-FISH in Lepidoptera and - as far as we know - in all insect. Because constructing a fosmid library is easier than a BAC library, FISH with fosmid clones will accelerate gene mapping in Lepidoptera.

Fosmid-FISH clearly revealed that gene order of S. c. ricini chromosomes 12 
and 13 was conserved in the corresponding parts of the neo-sex chromosomes of $S . c$. walkeri and S. cynthia subsp. indet. (Fig. 3). Consequently, the step-by-step evolution of the sex chromosome system as proposed by Yoshido et al. (2010) in the three S. cynthia subspecies has been fully confirmed in the present study. In addition, comparative gene mapping of their chromosomes shows a high degree of collinearity between the $S$. cynthia subspecies and B. mori (Fig. 3).

The supposed ancestral chromosome number in Lepidoptera is $n=31$ (Robinson, 1971; Lukhtanov, 2000; De Prins and Saitoh, 2003). B. mori $(n=28)$ is rather close to the ancestral chromosome number. We show here that the autosomes of $S$. cynthia are composed of either two or four B. mori homologues, apparently complete the karyotype, with one exception (see next paragraph). Thus the low number-karyotype of S. cynthia was indeed formed by multiple chromosome fusions. The same explanation was also proposed by Pringle et al. (2007) for the decreased chromosome number $(n=21)$ of the H. melpomene karyotype. The fusion partners, however, were different in the two species (Fig. 1, Table 1). Hence, the fusion events in S. cynthia have happened independently from those in H. melpomene.

The exceptional autosome, B. mori chromosome 11, appeared to have split to contribute to two S. cynthia chromosomes, S. c. ricini chromosome 11 and 12 . In fact, $B$. 
mori chromosome 11 itself is probably the fusion product of two separate ancestral chromosomes, as it was found homologous to two autosomes in yet another species, Manduca sexta (Yasukochi et al., 2009). Thus, S. c. ricini chromosome 11 and 12 are probably also the fusion products of two entire ancestral chromosomes each.

B. mori has a single NOR which is located in chromosome 11 (Yoshido et al., 2005a). According to our BAC-FISH mapping, the rDNA repeats in B. mori locate somewhere between $R p L 4$ (position $2.86 \mathrm{Mb}$ in Kaikobase) and Topo II (position 8.6 $\mathrm{Mb}$ in Kaikobase). Although a single NOR was also observed in S. c. ricini (Fig. 1), it maps to a region between the orthologs of B. mori RpL4 and P450 (in B. mori at position 2.86 $\mathrm{Mb}$ and 1.27 $\mathrm{Mb}$ of chromosome 11, respectively) (Table 1). The inverted order of RpL4 and NOR in S. c. ricini may be due to a rearrangement accompanying the fusion process or to NOR transposition. In $M$. sexta, the single NOR also had an altered position relative to B. mori (Yasukochi et al., 2009). Transposition or translocation of rDNA appears to be a rather common event in insects (Roy et al., 2005; Cabrero and Camacho, 2008; Nguyen et al., 2010) and plants (Schubert and Wobus, 1985; Dubcovsky and Dvoř́a, 1995; Datson and Murray, 2006).

Gene order is well conserved between B. mori chromosomes and the corresponding chromosome segments in S. cynthia. We detected no change in the order 
of orthologous genes in all cases in which the order could be inferred from mapping more than two probes (see Fig. 1, chromosome 5, 7, 12 and 13, and Table 1), the only exception being the rDNA locus. Gene-based comparative mapping has so far revealed that the gene order in B. mori $(\mathrm{n}=28)$ and either H. melpomene $(\mathrm{n}=21)$ (Pringle et al., 2007), B. anynana $(\mathrm{n}=28)$ (Beldade et al., 2009) or M. sexta $(\mathrm{n}=28)$ (Yasukochi et al., 2009) was rather well conserved too, with only a few translocations, inversions and fusion/fission events. In S. cynthia, even though the autosomes had undergone fusion events in their evolutionary history, gene order was conserved in the corresponding chromosome parts.

In contrast, the relationships of genomic components among dipteran species (fly and mosquito) based on genomic sequence resources have revealed higher degree of rearrangements. Comparative genome analysis in dipterans species, D. melanogaster, Anopheles gambiae, Aedes aegypti showed shuffling of gene order with only conservation of microsynteny among species (Zdobnov et al., 2002; Nene et al., 2007). Among more closely related species in genus Drosophila (12 species) or in genus Anopheles ( 2 species), their gene order has been also shuffled along corresponding chromosomal arms (Ranz et al., 2001; Sharakhov et al., 2002; Bhutkar et al., 2008). Taken together, in dipteran species, inter- and/or intra-chromosomal rearrangements 
may have been frequently occurred in evolutionary history. Hence, higher degree of internal stability in the lepidopteran chromosomes is rather surprising.

Both phenomena, conserved synteny of whole chromosomes and conserved gene order, were also observed in birds. Conserved gene order in the avian large autosomes, the so-called macrochromosomes, has been revealed by gene-based molecular linkage analysis (Backström et al., 2006, 2008; Stapley et al., 2008). Collinearity of the small chromosomes, the so-called microchromosomes, between chicken and turkey (Meleagris gallopavo) has also been detected (Griffin et al., 2008). A recent genome sequence comparison between chicken and zebra finch (Taeniopygia guttata) disclosed high homology between 6 chicken and 8 zebra finch macrochromosomes (Ellegren, 2010). Although inverted order of chromosomal segments and fusion/fission events are apparent between the distantly related bird species, the degree of synteny is high among birds. Ellegren (2010) attributed the stability of chromosomes to the lower density of interspersed repetitive elements in birds, approximately $10 \%$ in the chicken genome compared to $40-50 \%$ in other sequenced animal genomes. The lower density is supposed to reduce the possibility of chromosomal rearrangements mediated by repeat elements.

This explanation does not hold true for the conserved synteny and gene order in 
Lepidoptera. The B. mori genome contains $43.6 \%$ interspersed repetitive sequences (The International Silkworm Genome Consortium, 2008). There is also another reason to expect even more rearrangements than in other animals. The holokinetic chromosome structure of Lepidoptera (Murakami and Imai, 1974; Traut, 1986; Wolf, 1996) should stand more chromosomal rearrangements than the common monocentric structure (see discussion in Yasukochi et al., 2009). Since fragments of holokinetic chromosomes can survive during cell cycles (Fujiwara et al., 2000) or even several generations (Rathjens, 1974; Marec et al., 2001), the risk for lethality is much lower. It is the more surprising that Lepidoptera exhibit conserved synteny and high stability of gene order within chromosomes. It is open to which property of the genome or chromosome structure the Lepidoptera chromosomes owe their stability.

\section{Acknowledgements}

We thank W. Traut (Lübeck, Germany) for critical reading the manuscript and valuable suggestions. Our thanks also go to Y. Yamada (Sapporo, Japan) for supporting to rear Samia cynthia, T. Fujii (Tokyo, Japan) for providing S. c. ricini, and $\mathrm{M}$. 
Tanaka-Okuyama, H. Ohta, E. Igari, H. Hoshida (Tsukuba, Japan) for preparing fosmid library. A.Y. received a grant $19 \cdot 1114$ of Japan Society for the Promotion of Science (JSPS). We acknowledge the research fellowship 21-7147 given to K.S. from JSPS. 


\section{References}

Arunkumar, K.P., Tomar, A., Daimon, T., Shimada, T., Nagaraju, J., 2008. WildSilkbase: An EST database of wild silkmoths. BMC Genomics 9, 338.

Backström, N., Brandström, M., Gustafsson, L., Qvarnström, A., Cheng, H., Ellegren, H., 2006. Genetic mapping in a natural population of collared flycatchers (Ficedula albicollis): conserved synteny but gene order rearrangements on the avian $\mathrm{Z}$ chromosome. Genetics 174, 377-386.

Backström, N., Karaiskou, N., Leder, E.H., Gustafsson, L., Primmer, C.R., Qvarnström, A., Ellegren, H., 2008. A gene-based genetic linkage map of the collared flycatcher (Ficedula albicollis) reveals extensive synteny and gene-order conservation during 100 million years of avian evolution. Genetics 179, 1479-1495.

Beldade, P., Saenko, S.V., Pul, N., Long, A.D., 2009. A gene-based linkage map for Bicyclus anynana butterflies allows for a comprehensive analysis of synteny with the lepidopteran reference genome. PLoS Genet. 5, e1000366.

Bhutkar, A., Schaeffer, S.W., Russo, S.M., Xu, M., Smith, T.F., Gelbart, W.M., 2008. Chromosomal rearrangement inferred from comparison of 12 Drosophila genomes. Genetics 179, 1657-1680. 
Cabrero, J., Camacho, J.P.M., 2008. Location and expression of ribosomal RNA genes in grasshoppers: Abundance of silent and cryptic loci. Chromosome Res. 16, 595-607.

d'Alençon, E., Sezutsu, H., Legeai, F., Permal, E., Bernard-Samain, S., Gimenez, S., Gagneur, Z., et al., 2010. Extensive synteny conservation of holocentric chromosomes in Lepidoptera despite high rates of local genome rearrangements. Proc. Natl. Acad. Sci. USA 107, 7680-7685.

Dalzell, P., Miles, L.G., Isberg, S.R., Glenn, T.C., King, C., Murtagh, V., Moran, C., 2009. Standardized reference ideogram for physical mapping in the saltwater crocodile (Crocodylus porosus). Cytogenet. Genome Res. 127, 204-212.

Datson, P.M., Murray, B.G., 2006. Ribosomal DNA locus evolution in Nemesia: transposition rather than structural rearrangement as the key mechanism? Chromosome Res. 14, 845-857.

De Prins, J., Saitoh, K., 2003. Karyology and sex determination, in: Kristensen, N.P. (Ed.), Lepidoptera, moths and butterflies. 2. Morphology, physiology, and development. Walter de Gruyter, Berlin \& New York, pp. 449-468.

Dubcovsky, J., Dvořák, J., 1995. Ribosomal RNA multigene loci: nomads of the Triticeae genomes. Genetics 140, 1367-1377. 
Ellegren, H., 2010. Evolutionary stasis: the stable chromosomes of birds. Trends Ecol.

Evol. 25, 283-291.

Ferguson-Smith, M.A., Trifonov, V., 2007. Mammalian karyotype evolution. Nat. Rev.

Genet. 8, 950-962.

Fujiwara, H., Nakazato, Y., Okazaki, S., Ninaki, O., 2000. Stability and telomere

structure of chromosomal fragments in two different mosaic strains of the silkworm,

Bombyx mori. Zool. Sci. 17, $743-750$.

Griffin, D.K., Robertson, L.B., Tempest, H.G., Vignal, A., Fillon, V., Croolimans, R.P., et al., 2008. Whole genome comparative studies between chicken and turkey and their implications for avian genome evolution. BMC Genomics 9, 168.

Grimaldi, D.A., Engel, M.S., 2005. Evolution of the insects. Cambridge Univ. Press, New York.

Kristensen, N.P., Skalski, A.W., 1999. Phylogeny and palaeontology, in: Kristensen, N.P. (Ed.), Lepidoptera, moths and butterflies. 1. Evolution, systematics, and biogeography. Walter de Gruyter, Berlin \& New York, pp. 7-25.

Liu, C., Liu, J., Li, H., Zhang, Z., Han, Y., Huang, S., Jin, W., 2010. Karyotyping in melon (Cucumis melo L.) by cross-species fosmid fluorescence in situ hybridization. Cytogenet. Genome. Res. 129, 241-249. 
Lukhtanov, V.A., 2000. Sex chromatin and sex chromosome systems in non-ditrysian Lepidoptera (Insecta). J. Zool. Syst. Evol. Res. 38, 73-79.

Marec, F., Tothová, A., Sahara, K., Traut, W., 2001. Meiotic pairing of sex chromosome fragments and its relation to atypical transmission of a sex-linked marker in Ephestia kuehniella (Insecta: Lepidoptera). Heredity 87, 659-671.

Marec, F., Sahara, K., Traut, W., 2010. Rise and Fall of the W chromosome in Lepidoptera, in: Goldsmith, M.R., Marec, F. (Eds.), Molecular Biology and Genetics of the Lepidoptera. CRC Press, Boca Raton, FL, USA, pp. 49-63.

Murakami, A., Imai, H.T., 1974. Cytological evidence for holocentric chromosomes of the silkworms, Bomby mori and B. mandarina, (Bombycidae, Lepidoptera). Chromosoma 47, 167-178.

Nene, V., Wortman, J.R., Lawson, D., Haas, B., Kodira, C., Tu, Z.J., et al., 2007. Genome sequence of Aedes aegypti, a major arbovirus vector. Science 316, 1718-1723.

Nguyen, P., Sahara, K., Yoshido, A., Marec, F., 2010. Evolutionary dynamics of rDNA clusters on chromosomes of moths and butterflies (Lepidoptera). Genetica 138, 343-354.

Pringle, E.G., Baxter, S.W., Webster, C.L., Papanicolaou, A., Lee, S.F., Jiggins, C.D., 
2007. Synteny and chromosome evolution in the Lepidoptera: evidence from mapping in Heliconius melpomene. Genetics 177, 417-426.

Ranz, J.M., Casals, F., Ruiz, A., 2001. How malleable is the eukaryotic genome? Extreme rate of chromosomal rearrangement in the genus Drosophila. Genome Res. $11,230-239$.

Rathjens, B., 1974. Zur Funktion des W-chromatins bei Ephestia kuehniella (Lepidoptera). Isolierung und Charakterisierung von W-Chromatin-Mutanten. Chromosoma 47, 21-44.

Robinson, R., 1971. Lepidoptera genetics. Pergamon, Oxford.

Roy, V., Monti-Dedieu, L., Chaminade, N., Siljak-Yakovlev, S., Aulard, S., et al., 2005. Evolution of the chromosomal location of rDNA genes in two Drosophila species subgroups: ananassae and melanogaster. Heredity 94, 388-395.

Sahara, K., Marec, F., Traut, W., 1999. TTAGG telomeric repeats in chromosomes of some insects and other arthropods. Chromosome Res. 7, 449-460.

Sahara, K., Yoshido, A., Marec, F., Fuková, I., Zhang, H.B., Wu, C. C., et al., 2007. Conserved synteny of genes between chromosome 15 of Bombyx mori and a chromosome of Manduca sexta shown by five-color BAC-FISH. Genome 50, 1061-1065. 
Schubert, I., Wobus, U., 1985. In situ hybridization confirms jumping nucleolus organizing regions in Allium. Chromosoma 92, 143-148.

Sharakhov, I.V., Serazin, A.C., Grushko, O.G., Dana, A., Lobo, N., Hillenmeyer, M.E., et al., 2002. Inversions and gene order shuffling in Anopheles gambiae and A. funestus. Science 298, 182-185.

Shibata, F., Sahara, K., Naito, Y., Yasukochi, Y., 2009. Reprobing of multicolour FISH in preparations of lepidopteran chromosomes. Zool. Sci. 26, 187-190.

Stapley, J., Birkhead, T.R., Burke, T., Slate, J., 2008. A linkage map of the zebra finch Taeniopygia guttata provides new insights into avian genome evolution. Genetics $179,651-667$.

The International Silkworm Genome Consortium, 2008. The genome of a lepidopteran model insect, the silkworm Bombyx mori. Insect Biochem. Mol. Biol. 38, 1036-1045.

Traut, W., 1986. A genetic linkage study of W-chromosome-autosome fusions, breakage, and kinetic organization of chromosomes in Ephestia (Lepidoptera). Genetica 69, $69-79$.

Traut, W., Sahara, K., Marec, F., 2007. Sex chromosomes and sex determination in Lepidoptera. Sex. Dev. 1, 332-346.

Wolf, K.W., 1996. The structure of condensed chromosomes in mitosis and meiosis of 
insects. Int. J. Insect Morphol. Embryol. 25, 37-62.

Yasukochi, Y., 2002. PCR-based screening for bacterial artificial chromosome libraries. Methods Mol. Biol. 192, 401-410.

Yasukochi, Y., Ashakumary, L.A., Baba, K., Yoshido, A., Sahara, K., 2006. A second-generation integrated map of the silkworm reveals synteny and conserved gene order between lepidopteran insects. Genetics 173, 1319-1328.

Yasukochi, Y., Tanaka-Okuyama, M., Shibata, F., Yoshido, A., Marec, F., Wu, C., et al., 2009. Extensive conserved synteny of genes between the karyotypes of Manduca sexta and Bombyx mori revealed by BAC-FISH mapping. PLoS One 4, e7465.

Yoshido, A., Bando, H., Yasukochi, Y., Sahara, K., 2005a. The Bombyx mori karyotype and the assignment of linkage groups. Genetics 170, 675-685.

Yoshido, A., Marec, F., Sahara, K., 2005b. Resolution of sex chromosome constitution by genomic in situ hybridization and fluorescence in situ hybridization with (TTAGG)n telomeric probe in some species of Lepidoptera. Chromosoma 114, 193-202.

Yoshido, A., Sahara, K., Marec, F., Matsuda, Y., 2010. Step-by-step evolution of neo-sex chromosomes in geographical populations of wild silkmoths, Samia cynthia ssp. Heredity doi: 10.1038/hdy.2010.94. 
Zhang, L., Bao, Z., Wang, S., Hu, X., Hu, J., 2008. FISH mapping and identification of zhikong scallop (Chlamys farreri) chromosomes. Marine Biotechnol. 10, 151-157.

Zdobnov, E.M., von Mering, C., Letunic, I., Torrents, D., Suyama, M., Copley, R.R., Christophides, G.K., et al., 2002. Comparative genome and proteome analysis of Anopheles gambiae and Drosophila melanogaster. Science 298, 149-159. 


\section{Figure legends}

Fig. 1. FISH identification of individual pachytene bivalents in Samia cynthia ricini and their comparison with Bombyx mori chromosomes. Note that the lengths of chromosome bivalents cannot be compared in this figure as they are derived from different pachytene complements and/or preparations in both sexes. Vertical bars represent corresponding parts of $B$. mori chromosomes (black italic numbers) drawn to relative scales in Mb taken from Kaikobase. Samia chromosomes were counterstained with DAPI (light blue). For details of fosmid probes used and their labeling, see M\&M section, Table 1 and Table S2.

Fig. 2. Fosmid-FISH karyotype of Samia cynthia ricini. (a) A female postpachytene complement (most probably developing to a nurse cell) of 13 autosomal bivalents and a Z-chromosome univalent, each identified by 1-3 pseudocolored hybridization signals of fosmid probes. Chromosomes were counterstained with DAPI (light blue). A total of 22 fosmid were hybridized to this complement (for details, see Table 1 and Table S2). (b) Thirteen autosomal bivalents and the Z-chromosome univalent of the same postpachytene complement as in (a) arranged according to their chromosome numbers 
(see Table 1). Bar $=10 \mu \mathrm{m}$.

Fig. 3. Conserved synteny of genes between the sex chromosomes and its autosomal homologs in Samia cynthia subspecies. Signal orders of probes on the chromosome Z, 12 and 13 of S. c. ricini (drawing in the left side) are conserved both in an autosomal bivalent (a) and neo-sex chromosomes (b) of S. c. walkeri, and neo-sex chromosomes of S. cynthia subsp. indet. (c). Note that the distal end of the original Z chromosome parts in the neo-sex bivalent (b) and neo-sex trivalent (c), respectively, remained unpaired most probably because of length differences between paring partners. Also note the asymmetrical signals of each 32B23 (magenta) and 56J22 (red) fosmid probes on the S. cynthia subsp. indet. neo-sex chromosome trivalent, most probably resulting from unequal length of paired chromosomes. The sex chromosome system of each subspecies (populations) are described below the sex chromosome drawing and figures; $\mathrm{Z} 0$ for S. c. ricini (Vietnam), neo-Wneo-Z for S. c. walkeri (Sapporo) and neo- $\mathrm{WZ}_{1} \mathrm{Z}_{2}$ for S. cynthia subsp. indet. (Nagano). See Table S2 for details of probe information. 
Table 1 Summary of FISH mapping of Samia cynthia fosmids, containing orthologs of Bombyx mori genes; the fosmids are listed acccording to the order of corresponding hybridization signals in a particular chromosome (see Fig. 1).

\begin{tabular}{|c|c|c|c|c|c|c|}
\hline \multicolumn{5}{|c|}{ S. cynthia } & \multicolumn{2}{|r|}{ B. mori } \\
\hline Chr & fosmid & & & & $\mathrm{Chr}$ & Location in \\
\hline No. & code & Accession No. & EST (SilkBase*) & putative function & No. & Kaikobase** \\
\hline 1 & $39 \mathrm{~N} 17$ & DQ465407 & S06A01NCLL0005_L15 & 3-hydroxy-3-methylgultary-CoA reducase & 2 & $916,956-919,459$ \\
\hline 1 & 9G21 & & I10A02NGRL0003_G06 & ribosomal protein S21 & 2 & $9,846,943-9,848,321$ \\
\hline 1 & $28 \mathrm{O} 11$ & & S06A01NCLL0014_E20 & eukaryotic translation initiation factor $2 \alpha$ & 20 & $7,111,198-7,115,385$ \\
\hline 1 & $63 \mathrm{H} 6$ & & I10A02NGRL0008_J19 & ribosomal protein $\mathrm{S} 20$ & 20 & $13,287,125-13,290,285$ \\
\hline 1 & $57 \mathrm{~F} 7$ & & S13A02NGRL0004_B05 & hypothetical protein & 26 & $741,104-745,803$ \\
\hline 1 & $42 \mathrm{E} 11$ & & S13A01NGRL0005_O18 & THO complex subunit 3 & 26 & $2,003,467-2,008,338$ \\
\hline 1 & $62 \mathrm{D} 4$ & AB190810 & not found & vitellogenin & 27 & $10,593,279-10,604,046$ \\
\hline 1 & $21 \mathrm{~F} 6$ & & S13A02NGRL0008_K14 & HSP70 & 27 & $12,563,862-12,565,843$ \\
\hline 2 & $47 \mathrm{~N} 14$ & & S06A01NCLL0006_I20 & ribosomal protein L15 & 6 & $4,970,835-4,972,168$ \\
\hline 2 & $5 \mathrm{C} 24$ & & I09A02NGRL0003_D23 & attacin & 6 & $18,597,002-18,597,814$ \\
\hline 2 & $51 \mathrm{~F} 19$ & AB564748 & not found & laccase2 & 10 & $4,836,432-4,840,937$ \\
\hline 2 & $20 \mathrm{E} 12$ & & S13A01NGRL0005_E18 & diapause bioclock protein & 10 & $17,019,682-17,023,915$ \\
\hline 3 & $6 \mathrm{P} 17$ & & S13A01NGRL0008_G03 & serpin-4A & 28 & $10,271,213-10,272,445$ \\
\hline 3 & $35 \mathrm{P} 19$ & & I10A02NGRL0006_O06 & ribosomal protein S11 & 28 & $8,034,435-8,039,353$ \\
\hline 3 & $60 \mathrm{P} 13$ & & S13A01NGRL0009_H12 & ribosomal protein S27 & 7 & $8,032,934-8,033,836$ \\
\hline 3 & $55 \mathrm{M} 14$ & AB201280 & not found & chitinase & 7 & $14,117,862-14,119,517$ \\
\hline 4 & $20 \mathrm{~J} 22$ & & I10A02NGRL0006_L21 & cytoplasmic actin (A4) & 17 & $2,936,956-2,938,173$ \\
\hline 4 & $33 \mathrm{~F} 8$ & & I10A02NGRL0008_D04 & ribosomal protein S24 & 17 & $16,084,868-16,085,379$ \\
\hline 4 & $12 \mathrm{E} 18$ & & I09A02NGRL0002_J15 & ribosomal protein L19 & 5 & $3,573,371-3,577,651$ \\
\hline 4 & $25 \mathrm{G} 4$ & & I09A02NGRL0007_G07 & elongation factor $1 \alpha$ & 5 & $17,106,041-17,107,432$ \\
\hline 5 & $61 \mathrm{P} 10$ & & S06A01NCLL0022_K14 & ribosomal protein L5 & 15 & $5,494,680-5,499,312$ \\
\hline 5 & $63 \mathrm{~B} 20$ & & S06A01NCLL0009_G10 & ribosomal protein S5 & 15 & 7,586,991-7,587,650 \\
\hline 5 & $32 \mathrm{P} 7$ & & S13A01NGRL0001_M19 & ribosomal protein $\mathrm{S} 8$ & 15 & $13,998,126-14,000,450$ \\
\hline 5 & $27 \mathrm{~A} 12$ & & S13A02NGRL0011_B17 & bmtub4 & 15 & $16,664,500-16,666,892$ \\
\hline 5 & $18 \mathrm{O} 10$ & & S06A01NCLL0013_L06 & Ala-tRNA synthetase & 4 & $8,169,535-8,171,127$ \\
\hline 5 & $45 \mathrm{O} 20$ & AF015065 & S13A01NGRL0011_F03 & dopa decarboxylase & 4 & $12,504,927-12,521,471$ \\
\hline 6 & 29M1 & & S06A01NCLL0021_G23 & ribosomal protein L6 & 16 & $1,241,297-1,245,534$ \\
\hline 6 & $52 \mathrm{~K} 2$ & & I10A02NGRL0008_D12 & ribosomal protein $\mathrm{P} 2$ & 16 & $12,100,458-12,102,307$ \\
\hline 6 & $47 \mathrm{I} 5$ & & I09A02NGRL0007_E20 & ribosomal protein $\mathrm{S} 30$ & 23 & $12,462,114-12,465,725$ \\
\hline 6 & $56 \mathrm{E} 18$ & & S13A02NGRL0010_N13 & storage protein (SP1) & 23 & $16,906,448-16,911,216$ \\
\hline 7 & $44 \mathrm{~L} 5$ & & S06A01NCLL0024_M07 & elongation factor 1 gamma & 18 & $12,666,084-12,671,258$ \\
\hline 7 & 49D20 & AB026557 & not found & sensory neuron membrane protein-1 (snmp) & 18 & not mapped*** \\
\hline 7 & $13 \mathrm{~B} 10$ & & I09A02NGRL0002_I11 & ribosomal protein $\mathrm{S} 9$ & 19 & $1,216,844-1,218,990$ \\
\hline 7 & $52 \mathrm{I} 13$ & & S06A01NCLL0007_L10 & translation elongation factor 2 & 19 & $12,802,871-12,811,573$ \\
\hline 7 & $17 \mathrm{~F} 23$ & AB564749 & not found & egg-specific protein & 19 & $13,154,521-13,156,200$ \\
\hline 8 & $34 \mathrm{H} 14$ & L25668 & not found & PTTH & 22 & $10,390,124-10,391,823$ \\
\hline 8 & 12L19 & & I10A02NGRL0007_G03 & calreticulin & 22 & $4,685,110-4,689,288$ \\
\hline 8 & $23 \mathrm{~N} 7$ & & I09A02NGRL0004_J13 & ribosomal protein S25 & 25 & $6,334,666-6,349,673$ \\
\hline 8 & $46 \mathrm{C} 5$ & & S13A02NGRL0004_F13 & prophenoloxidase activating factor 3 & 25 & $8,534,344-8,540,906$ \\
\hline 9 & $59 \mathrm{~L} 14$ & & S13A02NGRL0001_I05 & arylphorin (SP2) & 3 & $8,650,251-8,654,561$ \\
\hline 9 & $52 \mathrm{~F} 14$ & AB564750 & not found & PKGIb & 3 & $8,060,428-8,060,994$ \\
\hline 9 & $55 \mathrm{G} 12$ & & I09A02NGRL0002_B18 & ribosomal protein L31 & 13 & $9,474,668-9,476,195$ \\
\hline 9 & 24K19 & & I09A02NGRL0007_H06 & ribosomal protein L21 & 13 & $16,214,502-16,216,987$ \\
\hline
\end{tabular}


Table 1 (continued)

\begin{tabular}{|c|c|c|c|c|c|c|}
\hline \multicolumn{5}{|c|}{ S. cynthia } & \multicolumn{2}{|r|}{ B. mori } \\
\hline Chr & fosmid & & & & Chr & Location in \\
\hline No. & code & Accession No. & EST (SikBase*) & putative function & No. & Kaikobase** \\
\hline 10 & $54 \mathrm{I} 24$ & & I09A02NGRL0001_C05 & allatostatin preprohormone & 14 & $205,746-208,559$ \\
\hline 10 & $26 \mathrm{D} 8$ & & S13A01NGRL0004_L17 & hypothetical protein & 14 & $14,055,940-14,059,769$ \\
\hline 10 & 53P19 & & S13A02NGRL0002_A22 & ribosomal protein S14 & 9 & $14,970,011-14,971,055$ \\
\hline 10 & $3 \mathrm{~A} 24$ & & I10A02NGRL0001_K04 & LCP18 & 9 & $18,065,584-18,066,900$ \\
\hline 11 & $11 \mathrm{P} 18$ & & S06A01NCLL0011_D13 & ADP/ATP translocase & 24 & $15,066,947-15,067,789$ \\
\hline 11 & $15 \mathrm{C} 21$ & AB220992 & I09A02NGRL0003_K24 & hemolin & 24 & $13,879,059-13,884,127$ \\
\hline 11 & $32 \mathrm{H} 9$ & & S06A01NCLL0008_E19 & $\mathrm{P} 450$ & 11 & $1,265,031-1,270,289$ \\
\hline 11 & not found & AB543313, AB543314 & S13A01NGRL0010_F15 & ribosomal protein L4 & 11 & $2,852,029-2,862,665$ \\
\hline 12 & $11 \mathrm{O} 1$ & AB543310 & not found & topoisomerase II (Topo II) & 11 & $8,589,427-8,602,332$ \\
\hline 12 & 44L2 & & S06A01NCLL0020_P12 & ribosomal protein L18 & 11 & $9,399,580-9,401,164$ \\
\hline 12 & 22B11 & & I09A02NGRL0005_B07 & ribosomal SOP2 & 11 & $19,584,552<<23,949,056^{* * *}$ \\
\hline 12 & $17 \mathrm{P9}$ & & S13A02NGRL0011_J07 & sorbitol dehydrogenase & 21 & $1,680,348-1,690,455$ \\
\hline 12 & 38013 & & S06A01NCLL0012_N21 & UBI3 mRNA for polyubiquitin & 21 & $11,614,170-11,616,911$ \\
\hline 13 & $56 \mathrm{~J} 8$ & & S13A01NGRL0004_C20 & hypothetical protein & 8 & $205,759-211,533$ \\
\hline 13 & $32 \mathrm{~B} 23$ & & S06A01NCLL0014_M19 & glycin rich protein & 8 & $4,927,144-4,928,289$ \\
\hline 13 & $56 \mathrm{~J} 22$ & AB564751 & not found & broad-complex (BR-C) & 8 & $18,077,270-18,081,471$ \\
\hline 13 & $75 \mathrm{O} 1$ & AB048258 & I10A02NGRL0003_P23 & lysozyme & 12 & $3,309,259-3,312,565$ \\
\hline 13 & $21 \mathrm{P} 14$ & & I10A02NGRL0003_B23 & p109 & 12 & $4,989,327-5,006,556$ \\
\hline 13 & 44E23 & & S13A02NGRL0001_G21 & $\mathrm{J}$ domain containing protein (JDCP) & 12 & $7,728,258-7,729,559$ \\
\hline 13 & $14 \mathrm{~J} 3$ & AB543315 & I09A02NGRL0003_I02 & xanthine dehydrogenase I (XDH I) & 12 & $8,493,509-8,511,254$ \\
\hline $\mathrm{Z}$ & $45 \mathrm{~A} 6$ & AB543309 & not found & kettin & 1 & $6,505,696-6,533,895$ \\
\hline $\mathrm{Z}$ & 19B8 & & S06A01NCLL0014_I05 & hypothetical protein & 1 & $11,424,630-11,431,079$ \\
\hline
\end{tabular}

*see http://silkbase.ab.a.u-tokyo.ac.jp/cgi-bin/index.cgi

**see http://kaikoblast.dna.affrc.go.jp/

*** The B. mori sensory neuron membrane protein-1 and ribosomal SOP2 mapped in the contigs 18 a and 11_3, respectively (Yasukochi et al., 2006). Only the location of ribosomal SOP2 can be determined from the primer sequence of the contigs 11_2 and 11_6 described in Tables S1 and S2 in Yasukochi et al. (2006). 

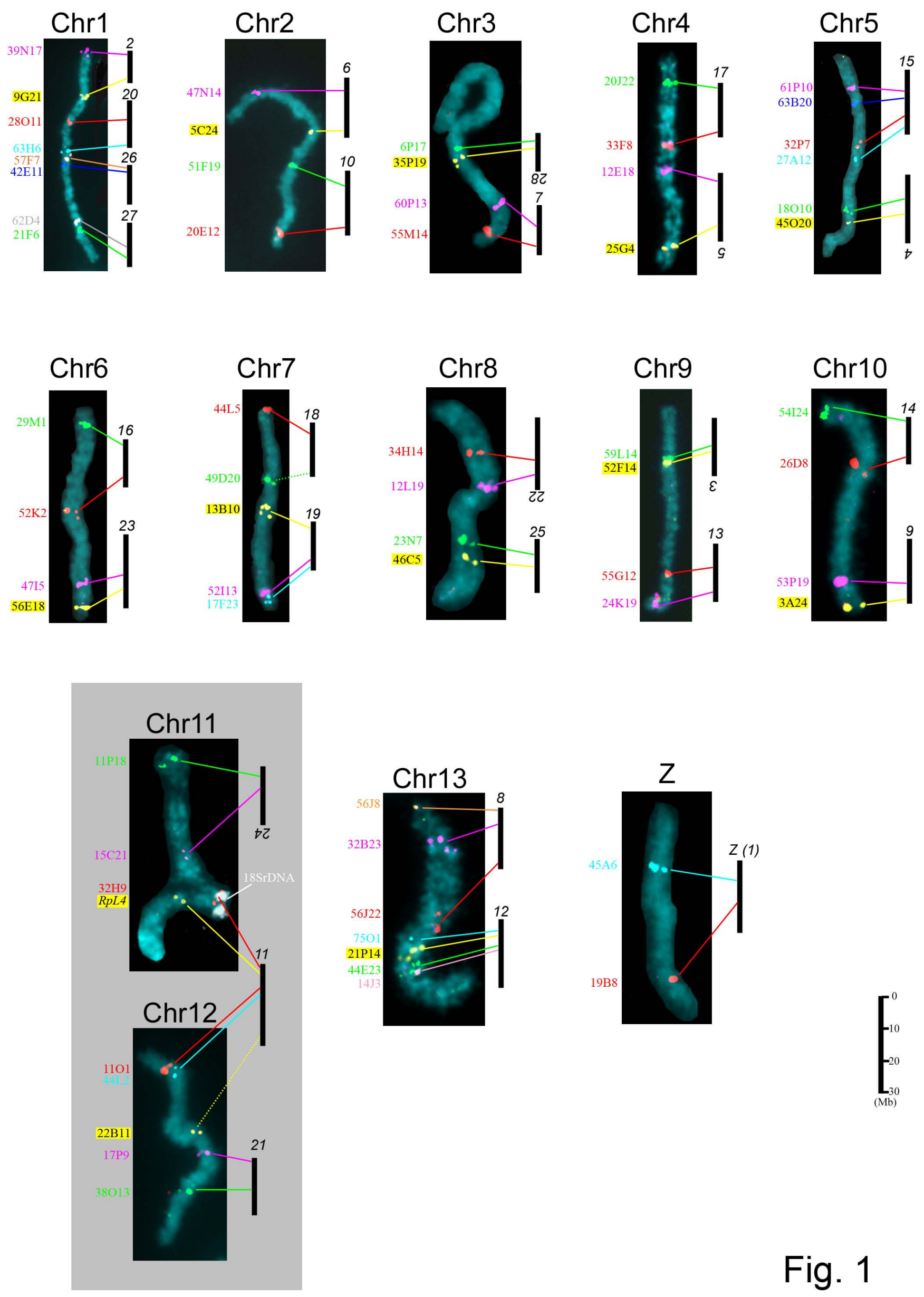

Fig. 1 
b
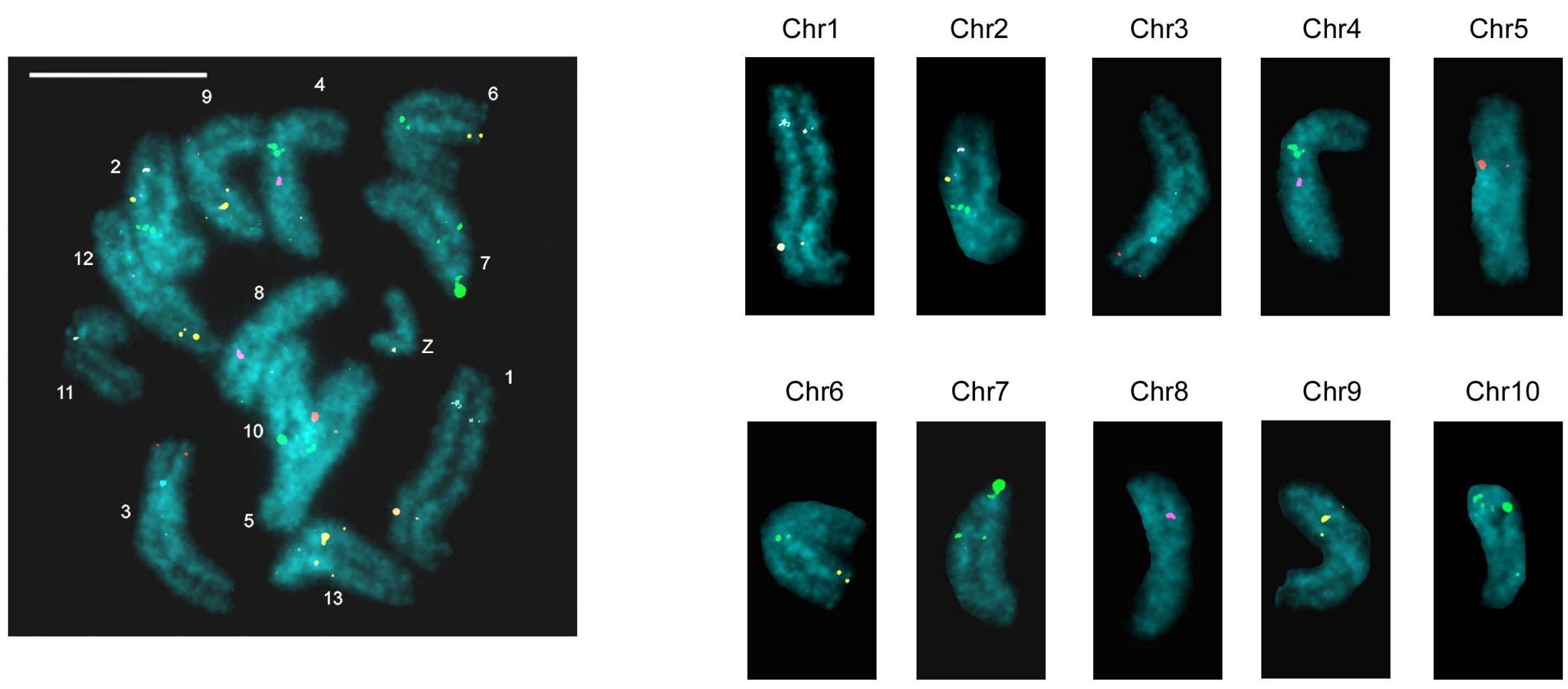

Chr10

Chr11
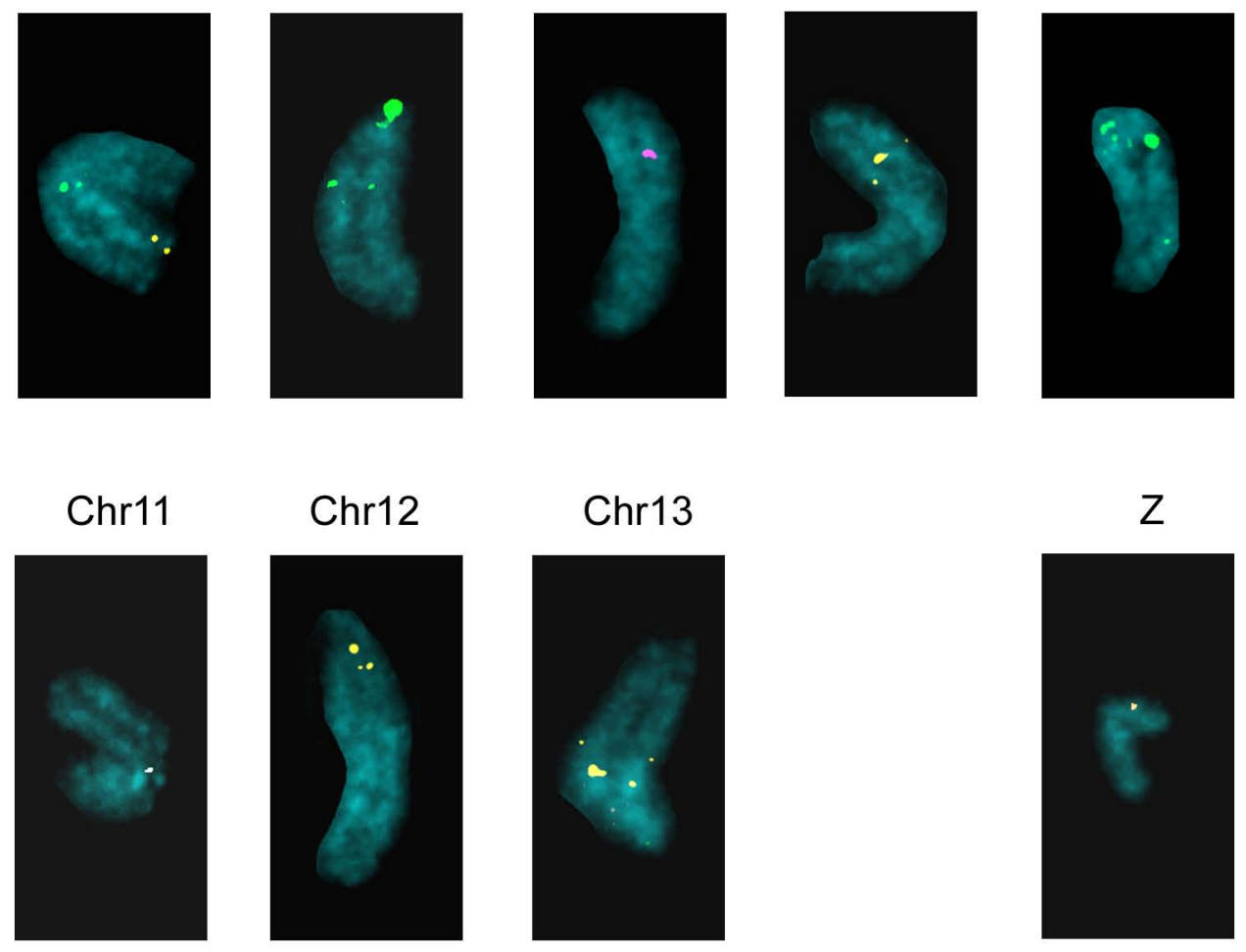

Z

Fig. 2

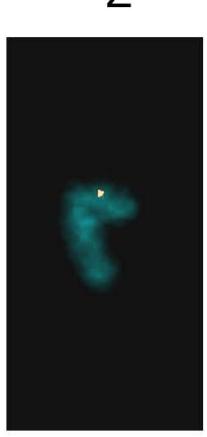




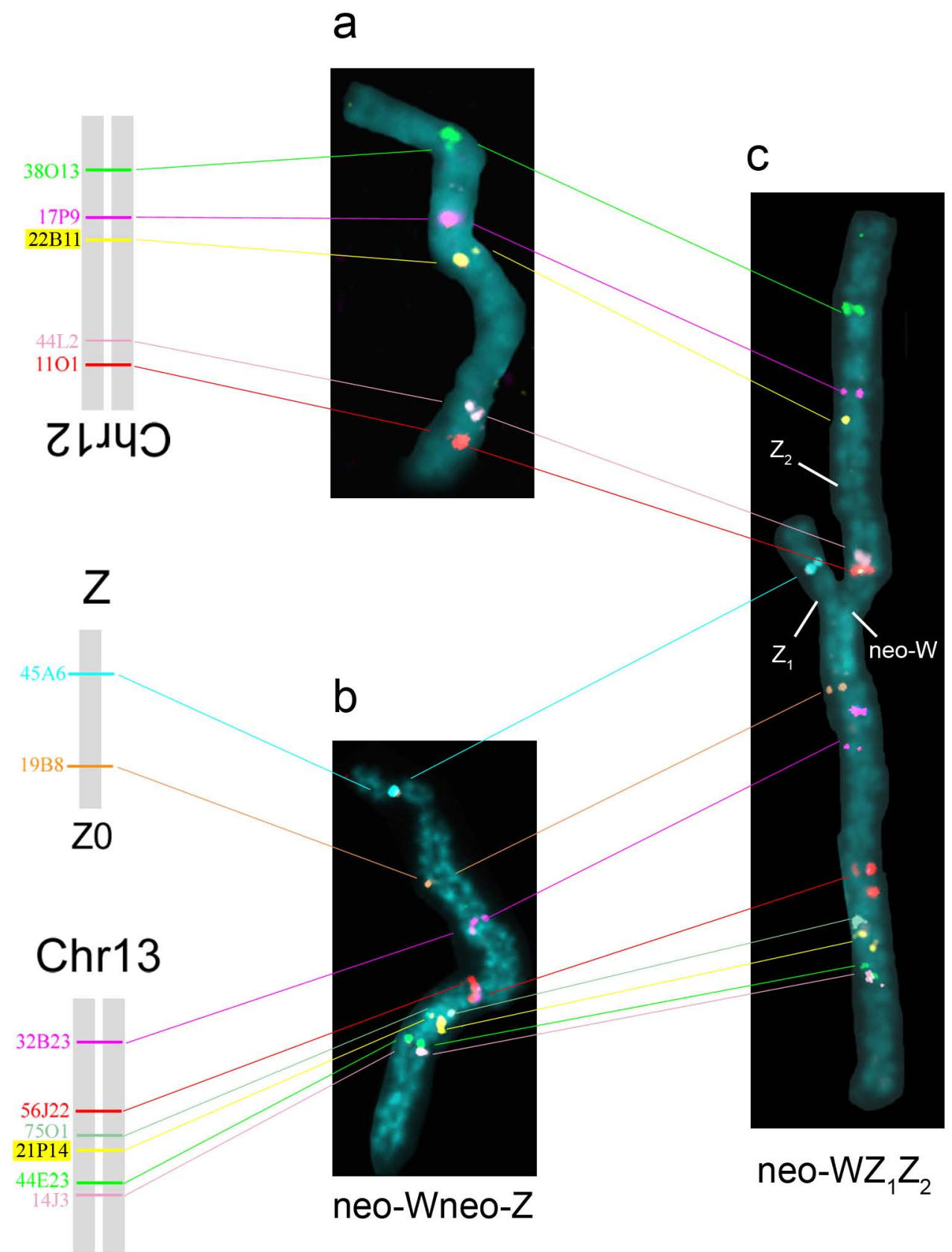

S. c. ricini

(Vietnam)
S. c. walkeri (Sapporo)
S. cynthia subsp. indet. (Nagano)

Fig. 3 